\title{
On the Number of Abelian Bordered Words
}

\author{
N. Rampersad ${ }^{1}$, M. Rigo ${ }^{2}$, and P. Salimov ${ }^{2,3 *}$ \\ 1 Dept. of Math. and Statistics, University of Winnipeg, 515 Portage Ave. Winnipeg, \\ $\mathrm{MB}, \mathrm{R} 3 \mathrm{~B}$ 2E9, Canada, narad.rampersad@gmail.com \\ 2 Dept of Math., University of Liège, Grande traverse 12 (B37), B-4000 Liège, \\ Belgium, M.Rigo@ulg. ac. be \\ 3 Sobolev Institute of Math., 4 Acad. Koptyug avenue, 630090 Novosibirsk, Russia.
}

\begin{abstract}
In the literature, many bijections between (labeled) Motzkin paths and various other combinatorial objects are studied. We consider abelian (un)bordered words and show the connection with irreducible symmetric Motzkin paths and paths in $\mathbb{Z}$ not returning to the origin. This study can be extended to abelian unbordered words over an arbitrary alphabet and we derive expressions to compute the number of these words. In particular, over a 3-letter alphabet, the connection with paths in the triangular lattice is made. Finally, we study the lengths of the abelian unbordered factors occurring in the Thue-Morse word.
\end{abstract}

\section{Introduction}

A finite word is bordered if it has a proper prefix that is also a suffix of the whole word. Otherwise, the word is said to be unbordered. Such properties have been investigated for a long time in combinatorics on words. For instance, the famous Duval's conjecture about the relationship between the length of a word and the maximum length of its unbordered factors has been solved in [8]. A classic result by Ehrenfeucht and Silberger [5] states that if an infinite word has only finitely many unbordered factors, then it is ultimately periodic.

Let us denote the Parikh vector of the word $u$ over $A$ by $\Psi(u)$ : i.e., $\Psi(u)$ is the element of $\mathbb{N}^{A}$ representing the number of occurrences of each letter in $u$. Two words $u$ and $v$ are abelian equivalent, if $\Psi(u)=\Psi(v)$. The notions of (un)bordered words are naturally extended to their abelian analogues by replacing equality with abelian equivalence. Such an extension is considered, for example, in [9].

This paper is organized as follows. Below, we make precise the basic definitions. In Section 2, we show that abelian unbordered words over a two letter alphabet are in one-to-one correspondence with particular Motzkin paths, i.e., lattice paths of $\mathbb{N}^{2}$ that begin at the origin, never pass below the $x$-axis, and use only up diagonal, down diagonal and horizontal steps. In Section 3, abelian unbordered words over a two letter alphabet are shown to be in one-to-one correspondence with $n$-step walks in $\mathbb{Z}$ starting from the origin but not returning

\footnotetext{
* The author is supported by the Russian President's grant no. MK-4075.2012.1, the Russian Foundation for Basic Research grant no. 12-01-00089 and by a University of Liège post-doctoral grant.
} 
to it. In particular, the number of these $n$-step walks is well-known and is given by the sequence A063886 in Sloane's Encyclopedia [12]. In Section 4, we extend the size of the alphabet and are still able to relate abelian unbordered words with specific paths and then derive a recursion formula to get the number of such words of length $n$. Interestingly, for a three letter alphabet, the connection is made with paths in the so-called triangular lattice. Finally, in Section 5, we consider the abelian unbordered factors occurring in abelian periodic automatic sequences (such as the Thue-Morse word). In this last section, we make use of Büchi's theorem and the formalism of first order logic as it was recently used in combinatorics on words, see for instance $[3,6]$.

Definition 1. A word $u \in A^{*}$ is abelian bordered if there exist $v, v^{\prime}, x, y \in A^{+}$ such that $u=v x=y v^{\prime}$ and $\Psi(v)=\Psi\left(v^{\prime}\right)$. In that case $v$ is an abelian border of $u$. Otherwise, $u$ is said to be abelian unbordered.

It is easy to see that if $u$ is abelian bordered, it has an abelian border of length at most $|u| / 2$.

Note that a word $u$ over $\{a, b\}$ is abelian unbordered if and only if its complement $\bar{u}$, where all $a$ 's are replaced with $b$ 's and all $b$ 's with $a$ 's, is also abelian unbordered. If a word is bordered, then it is trivially abelian bordered. But, in general, the converse does not hold. For instance, aabbabab is abelian bordered but not bordered.

Example 1. We consider the first few abelian unbordered words over $\{a, b\}$ that start with $a: a, a b, a a b, a b b, a a a b, a a b b, a b b b, a a a a b, a a a b b, a a b b b, a b b b b, a a b a b$, $a b a b b$. The first few values for the number of abelian unbordered words of length $n \geq 0$ over $\{a, b\}$ are: $2,2,4,6,12,20,40,70,140,252,504,924,1848,3432$, $6864, \ldots$ These values match Sloane's sequence A063886.

Remark 1. The language of abelian bordered words is not context-free. Indeed, if we intersect the language of abelian bordered words over $\{a, b\}$ with the regular language $a^{+} b^{+} a^{+} b^{+}$, then we get the language $\left\{a^{i} b^{j} a^{k} b^{\ell}: k \geq i\right.$ and $\left.j \geq \ell\right\}$. Using the pumping lemma, it is easy to show that this language is not contextfree.

\section{Connection with Motzkin Words}

The following is an immediate consequence of the definition "abelian unbordered".

Lemma 1. Let $n \geq 1$. A word $u_{1} \cdots u_{n} c v_{n} \cdots v_{1}$, where for all $i \in\{1, \ldots, n\}$, $u_{i} \in A, v_{i} \in A$, and $c \in\{\varepsilon\} \cup A$, is abelian unbordered if and only if, for all $i \in\{1, \ldots, n\}, \Psi\left(u_{1} \cdots u_{i}\right) \neq \Psi\left(v_{i} \cdots v_{1}\right)$.

Let us fix the alphabet $A=\{a, b\}$. If $x=x_{1} \cdots x_{n}$ and $y=y_{1} \cdots y_{n}$ are words of length $n$ over $A$, we define $\left(\begin{array}{l}x \\ y\end{array}\right) \in(A \times A)^{*}$ by

$$
\left(\begin{array}{l}
x \\
y
\end{array}\right):=\left(\begin{array}{l}
x_{1} \\
y_{1}
\end{array}\right) \cdots\left(\begin{array}{l}
x_{n} \\
y_{n}
\end{array}\right) \text {. }
$$


We also define the projection map

$$
\pi_{1}:(A \times A)^{*} \rightarrow A,\left(\begin{array}{l}
x \\
y
\end{array}\right) \mapsto x .
$$

We write $x^{R}$ to denote the reversal of $x$; that is, $x^{R}=x_{n} \cdots x_{1}$. We now define the map

$$
m: A^{*} \rightarrow(A \times A)^{*}, u \mapsto\left(\begin{array}{c}
u \\
u^{R}
\end{array}\right) .
$$

Let $P \subseteq(A \times A)^{*}$ be the context-free language $P=m\left(A^{*}\right)=\left\{\left(\begin{array}{c}u \\ u^{R}\end{array}\right) \mid u \in A^{*}\right\}$. Lemma 1 can be restated as follows.

Lemma 2. A word $u \in A^{+}$is abelian bordered if and only if there exists a nonempty proper prefix $p$ of $m(u)$ such that the numbers of occurrences of $\left(\begin{array}{l}a \\ b\end{array}\right)$ and $\left(\begin{array}{l}b \\ a\end{array}\right)$ in $p$ are the same.

Definition 2. A Grand Motzkin path of length $n$ is a lattice path of $\mathbb{N}^{2}$ running from $(0,0)$ to $(n, 0)$, whose permitted steps are the up diagonal step $(1,1)$, the down diagonal step $(1,-1)$ and the horizontal step $(1,0)$, called rise, fall and level step, respectively.

A Motzkin path is a Grand Motzkin path that never passes below the $x$-axis.

An irreducible (or elevated) Motzkin path is a Motzkin path that does not touch the $x$-axis except for the origin and the final destination [1].

If the level steps are labeled by $k$ colors (here colors will be letters from the alphabet A) we obtain a $k$-colored Motzkin path [11]. A $k$-colored Motzkin path is described by a word over the alphabet $\left\{R, F, L_{1}, \ldots, L_{k}\right\}$ and the context-free language of the $k$-colored Motzkin paths is denoted by $\mathcal{M}_{k}$. In particular, a Motzkin path described by a word over $\{R, F\}$ is a Dyck path.

Let $h:(A \times A)^{*} \rightarrow\left\{R, F, L_{a}, L_{b}\right\}^{*}$ be the coding

$$
h:\left(\begin{array}{l}
a \\
b
\end{array}\right) \mapsto R,\left(\begin{array}{l}
b \\
a
\end{array}\right) \mapsto F,\left(\begin{array}{l}
a \\
a
\end{array}\right) \mapsto L_{a},\left(\begin{array}{l}
b \\
b
\end{array}\right) \mapsto L_{b} .
$$

Note that if $p$ belongs to $P$, then $h(p)$ is a symmetric Grand Motzkin path having a symmetry axis $x=n / 2$. Let $\iota:\left\{R, F, L_{a}, L_{b}\right\}^{*} \rightarrow\left\{R, F, L_{a}, L_{b}\right\}^{*}$ defined by $\iota(R)=F, \iota(F)=R, \iota\left(L_{a}\right)=L_{a}$ and $\iota\left(L_{b}\right)=L_{b}$. If $w$ is a word over $\left\{R, F, L_{a}, L_{b}\right\}$, then $\widetilde{w}=\iota\left(w^{R}\right)$. A symmetric Grand Motzkin path is described by a word of the kind $w c \widetilde{w}$ where $c \in\left\{\varepsilon, L_{a}, L_{b}\right\}$.

Example 2. Two Motzkin paths colored with the letters $a$ and $b$ are represented in Figure 1. The left one is described by the word $R L_{a} R F R L_{b} F F R F$ and the right one is symmetric and irreducible. It corresponds to the word $h(m(a a a a b a b a b a b b))=R R L_{a} R F R F R F L_{a} F F$. 


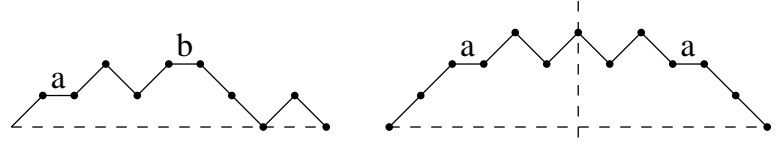

Fig. 1. Two Motzkin paths.

Lemma 3. A word u starting with a is abelian unbordered if and only if $h(m(u))$ is a symmetric and irreducible Motzkin path.

Proof. This is a reformulation of Lemma 2.

Proposition 1. The set of abelian unbordered words over $\{a, b\}$ starting with a and of length at least 2 is a $\pi_{1}\left(P \cap h^{-1}\left(\mathcal{M}_{2}\right)\right) b$.

Proof. Note that $h\left(P \cap h^{-1}\left(\mathcal{M}_{2}\right)\right)$ is the set of all symmetric 2-colored Motzkin paths. Now observe that if $u$ belongs to $a \pi_{1}\left(P \cap h^{-1}\left(\mathcal{M}_{2}\right)\right) b$, then $h(m(u))$ starts with $R$ and ends with $F$. So the corresponding 2-colored Motzkin path is irreducible. Conversely, if $u$ is abelian unbordered and starts with $a$, then by Lemma 3, $h(m(u))=R M F$, where $M$ is a symmetric 2-colored Motzkin path. Thus, $u \in a \pi_{1}\left(P \cap h^{-1}\left(\mathcal{M}_{2}\right)\right) b$.

Remark 2. Any symmetric 2-colored Motzkin path can be built by reflecting a prefix of a 2-colored Motzkin path. Let $w \in\left\{R, F, L_{a}, L_{b}\right\}^{*}$ be a prefix of length $k-1$ of a word in $\mathcal{M}_{2}$. By the previous proposition, we get that $a \pi_{1}\left[h^{-1}(w \widetilde{w})\right] b$, $a \pi_{1}\left[h^{-1}\left(w L_{a} \widetilde{w}\right)\right] b$ and $a \pi_{1}\left[h^{-1}\left(w L_{b} \widetilde{w}\right)\right] b$ are respectively an abelian unbordered word of length $2 k$, of length $2 k+1$ having $a$ as central letter, of length $2 k+1$ having $b$ as central letter.

- The set of abelian unbordered words of length $2 k$ starting with $a$ is in oneto-one correspondence with the set of prefixes of length $k-1$ of words in $\mathcal{M}_{2}$. Equivalently, the set of abelian unbordered words of length $2 k$ starting with $a$ is in one-to-one correspondence with the set of prefixes of length $k$ of irreducible 2-colored Motzkin paths.

- The set of abelian unbordered words of length $2 k+1$ starting with $a$ and having a central letter equal to $a$ (resp. $b$ ) is in one-to-one correspondence with the set of prefixes of length $k-1$ of words in $\mathcal{M}_{2}$. Equivalently, the set of abelian unbordered words of length $2 k+1$ starting with $a$ is in one-to-one correspondence with the set of prefixes of length $k$ of irreducible 2-colored Motzkin paths.

\section{Connection with the Sequence A063886}

The sequence A063886 gives the number $s(n)$ of $n$-step walks in $\mathbb{Z}$ starting from the origin but not returning to it. Such walks can be described by words over $\{r, \ell\}$ for right and left steps. The aim of this section is to show that the set of 
abelian unbordered words over a binary alphabet is in one-to-one correspondence with the $n$-step walks in $\mathbb{Z}$ starting from the origin but not returning to it. Let us first collect some well-known facts. The generating function for $s(n)$ is $\sqrt{\frac{1+2 x}{1-2 x}}$.

Consider a word $w=u_{1} \cdots u_{n} v_{n} \cdots v_{1} \in\{a, b\}^{*}$ of length $2 n$. We consider the map $c$ by

$$
c:\left(\begin{array}{l}
a \\
b
\end{array}\right) \mapsto r r, \quad\left(\begin{array}{l}
b \\
a
\end{array}\right) \mapsto \ell \ell, \quad\left(\begin{array}{l}
a \\
a
\end{array}\right) \mapsto \ell r, \quad\left(\begin{array}{l}
b \\
b
\end{array}\right) \mapsto r \ell
$$

Applying $c$ to the prefix of length $n$ of $m(w)$ provides a unique path of length $2 n$ in $\mathbb{Z}$. This path is denoted by $\mathfrak{p}(w)$. It is clear that $\mathfrak{p}$ is a one-to-one correspondence between the words of length $2 n$ over $\{a, b\}$ and the paths of length $2 n$ in $\mathbb{Z}$ starting from the origin. The following proposition follows immediately from Lemma 2.

Proposition 2. A word $w$ over $\{a, b\}$ of even length is abelian unbordered if and only if the path $\mathfrak{p}(w)$ does not return to the origin.

We extend the definition of $\mathfrak{p}$ to words of odd length by

$$
\mathfrak{p}\left(u_{1} \cdots u_{n} \alpha v_{n} \cdots v_{1}\right)=\left\{\begin{array}{l}
\mathfrak{p}\left(u_{1} \cdots u_{n} v_{n} \cdots v_{1}\right) \ell, \text { if } \alpha=a \\
\mathfrak{p}\left(u_{1} \cdots u_{n} v_{n} \cdots v_{1}\right) r, \text { if } \alpha=b
\end{array}\right.
$$

With this definition, $\mathfrak{p}$ is a one-to-one correspondence between the abelian unbordered words of length $2 n+1$ over $\{a, b\}$ and the paths of length $2 n+1$ in $\mathbb{Z}$ starting from the origin and not returning to it. It is therefore easy to get a result similar to the above proposition for words of odd length.

Proposition 3. A word $w$ over $\{a, b\}$ of odd length is abelian unbordered if and only if the path $\mathfrak{p}(w)$ does not return to the origin.

The number of prefixes of Motzkin paths is well-known [10, Theorem 1]. Here, we have obtained the following.

Corollary 1. The number of prefixes of length $k$ of 2-colored Motzkin paths is equal to half the number of paths in $\mathbb{Z}$ of length $2 k+2$ starting from the origin but not returning to it. In particular, this number is equal to

$$
\frac{1}{2}\left(\begin{array}{c}
2 k+2 \\
k+1
\end{array}\right)
$$

\section{Larger Alphabets}

Let $k \geq 2$. Consider the alphabet $A=\left\{a_{1}, \ldots, a_{k}\right\}$, or simply $\{1, \ldots, k\}$, and $\mathbb{Z}^{k}$ equipped with the usual unit vectors $\mathbf{e}_{1}, \ldots, \mathbf{e}_{k}$, whose coordinates are all equal to zero except one which is equal to 1 . To be able to define $k$-colored paths, we assume that at each point in $\mathbb{Z}^{k}$, there are exactly $k$ loops colored with the $k$ different letters. 
We first consider a word $u_{1} \cdots u_{n} v_{n} \cdots v_{1}$ of even length $2 n$. Take the prefix of length $n$ of $m\left(u_{1} \cdots u_{n} v_{n} \cdots v_{1}\right)$ and apply to it the morphism $h_{k}:(A \times A)^{*} \rightarrow$ $\left\{\mathbf{e}_{i}-\mathbf{e}_{j} \mid 1 \leq i, j \leq k\right\}^{*} \subset\left(\mathbb{Z}^{k}\right)^{*}$ defined by

$$
h_{k}\left(\begin{array}{c}
a_{i} \\
a_{j}
\end{array}\right)=\mathbf{e}_{i}-\mathbf{e}_{j}, \quad \forall i, j \in\{1, \ldots, k\} .
$$

Therefore, to the word $w=u_{1} \cdots u_{n} v_{n} \cdots v_{1}$ there corresponds the sequence of $n+1$ points in $\mathbb{Z}^{k}$

$$
p_{0}=\mathbf{0}, p_{1}=h_{k}\left(\begin{array}{l}
u_{1} \\
v_{1}
\end{array}\right), p_{2}=h_{k}\left(\begin{array}{l}
u_{1} \\
v_{1}
\end{array}\right)+h_{k}\left(\begin{array}{l}
u_{2} \\
v_{2}
\end{array}\right), \ldots, p_{n}=\sum_{j=1}^{n} h_{k}\left(\begin{array}{l}
u_{j} \\
v_{j}
\end{array}\right)
$$

where $\mathbf{0}$ denotes the origin $(0,0, \ldots, 0)$. By the definition of $h_{k}$, note that all these points lie in the subspace $\mathbf{H}_{k}$ of $\mathbb{Z}^{k}$ satisfying the equation

$$
x_{1}+\cdots+x_{k}=0 \text {. }
$$

Definition 3. A path of length $n$ in $\mathbf{H}_{k}$ is a sequence $p_{0}, \ldots, p_{n}$ of points in $\mathbf{H}_{k}$ such that, for all $j \geq 1, p_{j}-p_{j-1}$ belongs to $\left\{\mathbf{e}_{i}-\mathbf{e}_{j} \mid 1 \leq i, j \leq k, i \neq j\right\}$.

$A k$-colored path of length $n$ in $\mathbf{H}_{k}$ is a sequence $p_{0}, c_{0}, p_{1}, c_{1}, \ldots, p_{n-1}, c_{n-1}$, $p_{n}$ alternating points in $\mathbf{H}_{k}$ and elements belonging to $A \cup\{\varepsilon\}$ in such a way that, if $p_{j} \neq p_{j+1}$, then $p_{j+1}-p_{j}$ belongs to $\left\{\mathbf{e}_{i}-\mathbf{e}_{j} \mid 1 \leq i, j \leq k, i \neq j\right\}$ and $c_{j}=\varepsilon$, otherwise $c_{j}$ belongs to $A$ and can be interpreted as the color assigned to a loop on $p_{j}$. Note that paths are special cases of $k$-colored paths.

For the rest of this paper we will only consider paths that start at the origin.

Remark 3. For $k=3, \mathbf{H}_{3}$ corresponds to the so-called triangular lattice (sometimes called hexagonal lattice) because a point $\mathbf{x}$ has exactly six neighbors. The set of neighbors of $\mathbf{x}$ is denoted by

$$
N(\mathbf{x}):=\mathbf{x}+\left\{\mathbf{e}_{1}-\mathbf{e}_{2}, \mathbf{e}_{1}-\mathbf{e}_{3}, \mathbf{e}_{2}-\mathbf{e}_{1}, \mathbf{e}_{2}-\mathbf{e}_{3}, \mathbf{e}_{3}-\mathbf{e}_{1}, \mathbf{e}_{3}-\mathbf{e}_{2}\right\} .
$$

Consider the word $w=23321211$ over the alphabet $\{1,2,3\}$. The prefix of length 4 of $m(w)$ is

$$
\left(\begin{array}{llll}
2 & 3 & 3 & 2 \\
1 & 1 & 2 & 1
\end{array}\right)
$$

and corresponds to the sequence of moves $p_{1}-0=\mathbf{e}_{2}-\mathbf{e}_{1}, p_{2}-p_{1}=\mathbf{e}_{3}-\mathbf{e}_{1}$, $p_{3}-p_{2}=\mathbf{e}_{3}-\mathbf{e}_{2}$ and $p_{4}-p_{3}=\mathbf{e}_{2}-\mathbf{e}_{1}$ and the path represented in Fig. 2. The second path in Fig. 2 is colored and has four loops with labels 2, 1,3 and 1 respectively. It corresponds to $w^{\prime}=2321323113121211$. The prefix of length 8 of $m\left(w^{\prime}\right)$ is

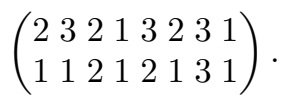

Observe that in this prefix we have an occurrence of a repeated symbol in positions 3,4 and 7,8 corresponding to the four loops in the path. 

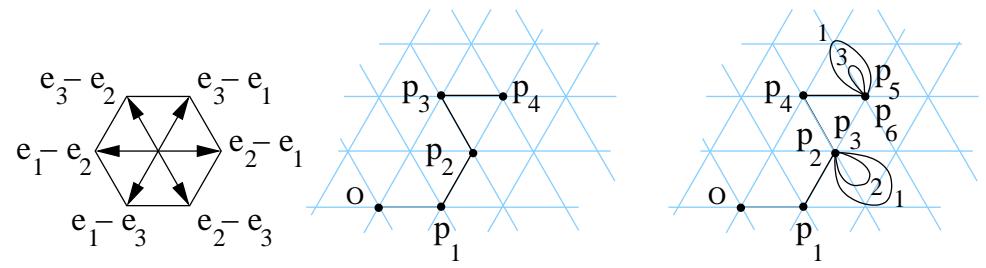

Fig. 2. In the triangular lattice, a path and a 3-colored path.

The word $w=u_{1} \cdots u_{n} v_{n} \cdots v_{1}$ is said to be simple if, for all $i \in\{1, \ldots, n\}$, $u_{i} \neq v_{i}$. In this case, in the sequence of points $p_{0}=\mathbf{0}, p_{1}, \ldots, p_{n}$ corresponding to $w$, for all $j<n$, we have $p_{j} \neq p_{j+1}$. Therefore simple words $w$ of length $2 n$ correspond to paths of length $n$ in $\mathbf{H}_{k}$. Such paths are denoted by $\mathfrak{p}(w)$. For a non-simple word $w$ of length $2 n$ there is a corresponding $k$-colored path $\mathfrak{p}(w)$ of length $n$ in $\mathbf{H}_{k}$ (where at least one loop $p_{j}=p_{j+1}$ occurs along the path). Conversely, for each $k$-colored path of length $n$ in $\mathbf{H}_{k}$, there is a corresponding word of length $2 n$.

Proposition 4. A word $w$ over $\left\{a_{1}, \ldots, a_{k}\right\}$ of even length $2 n$ is abelian unbordered if and only if the $k$-colored path $\mathfrak{p}(w)$ in $\mathbf{H}_{k}$ of length $n$ does not return to the origin. Moreover, a simple word $w$ over $\left\{a_{1}, \ldots, a_{k}\right\}$ of even length $2 n$ is abelian unbordered if and only if $\mathfrak{p}(w)$ is a path in $\mathbf{H}_{k}$ of length $n$ without loops that does not return to the origin.

Proof. The proof is similar to the one of Proposition 2.

If $w=u_{1} \cdots u_{n} \alpha v_{n} \cdots v_{1}$ is a word of odd length, we can first consider the prefix of length $n$ of $m(w)$ and then add an extra loop of color $\alpha$ to the end of the corresponding path $\mathfrak{p}(w)$. As for Proposition 3, we get the following.

Proposition 5. A word $w$ over $\left\{a_{1}, \ldots, a_{k}\right\}$ of odd length $2 n+1$ is abelian unbordered if and only if the $k$-colored path $\mathfrak{p}(w)$ of length $n+1$ in $\mathbf{H}_{k}$ does not return to the origin. In particular, such a path ends with a loop whose color is the one corresponding to the central letter of $w$.

Remark 4. The numbers of abelian unbordered words of length $n$ over a 3-letter alphabet, for $1 \leq n \leq 10$, are: 3, 6, 18, 48, 144, 402, 1206, 3438, 10314, 29754 and for simple abelian unbordered words, we get 3, 6, 18, 30, 90, 168, 504, 954, 2862,5508 . As we can observe, over a 3-letter alphabet, the number of abelian unbordered words (resp. simple abelian unbordered words) of length $2 n+1$ is three times the number of abelian unbordered words (resp. simple abelian unbordered words) of length $2 n$ because there are three available choices for the central letter. This observation extends trivially to an arbitrary alphabet.

From the discussion above and taking only entries of even index in the previous table, we also get the number of paths (resp. paths without loops) of length $n$ in the triangular lattice $\mathbf{H}_{3}$ that do not return to the origin. We denote this 
quantity by $p_{3}(n)$ (resp. $\left.s_{3}(n)\right)$. The first few values of $p_{3}(n), n \geq 1$, are 6,48 , $402,3438,29754,259464,2274462$ and the first few values of $s_{3}(n)$ are 6,30 , $168,954,5508,32016,187200$. The next statement means that one only needs to compute the sequence $\left(s_{k}(n)\right)_{n \geq 1}$ to get $\left(p_{k}(n)\right)_{n \geq 1}$ and thus the number of abelian unbordered words of length $n$.

Lemma 4. We have

$$
p_{k}(n)=\sum_{i=1}^{n} s_{k}(i) k^{n-i}\left(\begin{array}{l}
n-1 \\
n-i
\end{array}\right) .
$$

Proof. By a ( $k$-colored) path, we mean a path in $\mathbf{H}_{k}$ that does not return to the origin. Each such $k$-colored path of length $n$ has a unique underlying path of length $i$, for some $i \in\{1, \ldots, n\}$. To get a $k$-colored path of length $n, n-i$ loops are added to this underlying path. Each loop can be placed independently at any point of the path, except the origin, and can be colored independently in one of $k$ colors. So, the total number of ways to extend such a path of length $i$ to a $k$-colored path of length $n$ is $k^{n-i}\left(\begin{array}{c}n-1 \\ n-i\end{array}\right)$.

\subsection{Computation of $\left(s_{3}(n)\right)_{n \geq 0}$ and then $\left(s_{k}(n)\right)_{n \geq 0}$}

We show how to get a recurrence relation to compute the number $s_{3}(n)$, i.e., the number of paths in the triangular lattice $\mathbf{H}_{3}=(V, E)$ that do not return to the origin; here $V$ (resp. $E$ ) is the set of vertices (resp. edges) of $\mathbf{H}_{3}$. Consider the map

$$
e: V \rightarrow \mathbb{N}, \quad \mathbf{x} \mapsto \begin{cases}1 & \text { if } \mathbf{x}=\mathbf{0}, \\ 0 & \text { otherwise }\end{cases}
$$

If $f: V \rightarrow \mathbb{N}$ is a map, we denote by $\mathcal{S} f: V \rightarrow \mathbb{N}$ the map defined by

$$
(\mathcal{S} f)(\mathbf{x})=\sum_{\mathbf{y} \in N(\mathbf{x})} f(\mathbf{y})
$$

where $N(\mathbf{x})$ is the set of neighbors of $\mathbf{x}$. In particular, if $f, g: V \rightarrow \mathbb{N}$ are maps, then $\mathcal{S}(f+g)=\mathcal{S} f+\mathcal{S} g$. A simple induction argument gives the following result.

Lemma 5. With the above notation, $\left(\mathcal{S}^{n} e\right)(\mathbf{x})$ is equal to the number of paths of length $n$ that end at $\mathbf{x}$.

The values of the maps $e, \mathcal{S} e$ and $\mathcal{S}^{2} e$ around $\mathbf{0}$ are given in Figure 3 . Let $r_{3, n}: V \rightarrow \mathbb{N}$ be defined as follows: $r_{3, n}(\mathbf{x})$ is the number of paths of length $n$ that end at $\mathbf{x}$ and never return to the origin. Then

$$
s_{3}(n)=\sum_{\mathbf{x} \in V} r_{3, n}(\mathbf{x})
$$

where the sum is finite, since $r_{3, n}(\mathbf{x}) \neq 0$ implies that $\mathbf{x}$ is at distance at most $n$ from the origin. If a map $f: V \rightarrow \mathbb{N}$ is constant on $N(\mathbf{0})$ (as is the case for 


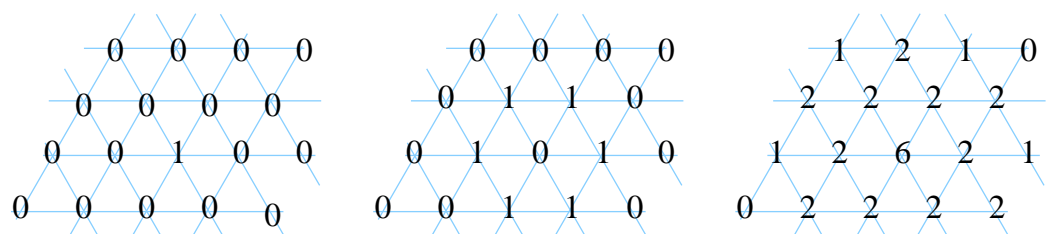

Fig. 3. values of $e, \mathcal{S} e$ and $\mathcal{S}^{2} e$ around $\mathbf{0 .}$

$\left.\mathcal{S}^{n} e\right)$, then $\gamma(f)$ is a shorthand for $f(\mathbf{y})$ for any $\mathbf{y} \in N(\mathbf{0})$. By the symmetry of $\mathbf{H}_{3}$, we see that $r_{3, n}$ is constant on $N(\mathbf{0})$. Note that

$$
s_{3}(n+1)=6 s_{3}(n)-\sum_{\mathbf{x} \in N(\mathbf{0})} r_{3, n}(\mathbf{x})=6 s_{3}(n)-6 \gamma\left(r_{3, n}\right)
$$

because all paths except the ones that end in vertices adjacent to 0 have 6 prolongations, and the excluded ones have 5 possible prolongations. The same argument can be applied to maps: $r_{3, n+1}=\mathcal{S} r_{3, n}-6 \gamma\left(r_{3, n}\right) e$ and, applied inductively, this leads to the following relation for $r_{3, n+1}$ :

$$
r_{3, n+1}=\mathcal{S}^{n+1} e-\sum_{i=0}^{n} 6 \gamma\left(r_{3, i}\right) \mathcal{S}^{n-i} e .
$$

The sequence $\left(\left(\mathcal{S}^{n} e\right)(\mathbf{0})\right)_{n \geq 0}$ counting the paths of length $n$ starting and ending at $\mathbf{0}$ is well-known (A002898 gives the number of $n$-step closed paths on the hexagonal lattice). For instance, we have

$$
\left(\mathcal{S}^{n} e\right)(\mathbf{0})=\sum_{\ell=0}^{n}(-2)^{n-\ell}\left(\begin{array}{l}
n \\
\ell
\end{array}\right) \sum_{j=0}^{\ell}\left(\begin{array}{l}
\ell \\
j
\end{array}\right)^{3}
$$

and its first values are 1, 0, 6, 12, 90, 360, 2040, 10080, 54810, 290640,... Due to the 6 -fold symmetry of the maps around the origin, note that

$$
\gamma\left(\mathcal{S}^{n} e\right)=\frac{\left(\mathcal{S}^{n+1} e\right)(\mathbf{0})}{6}
$$

Taking into account both (1) and (3), for all $n \geq 0$, we have

$$
\gamma\left(r_{3, n+1}\right)=\frac{\left(\mathcal{S}^{n+2} e\right)(\mathbf{0})}{6}-\sum_{i=0}^{n} \gamma\left(r_{3, i}\right)\left(\mathcal{S}^{n-i+1} e\right)(\mathbf{0})
$$

and $\gamma\left(r_{3,0}\right)=0$. As a conclusion, using the sequence $\left(\left(\mathcal{S}^{n} e\right)(\mathbf{0})\right)_{n \geq 0}$, we can compute inductively $\left(\gamma\left(r_{3, n}\right)\right)_{n \geq 0}$ and therefore $\left(s_{3}(n)\right)_{n \geq 0}$. Using the above formula, the first values of $\left(\gamma\left(r_{3, n}\right)\right)_{n \geq 0}$ are $0,1,2,9,36,172,816,4101,20840,108558$, $572028, \ldots$ Knowing that $s_{3}(0)=1$ is enough to compute

$$
s_{3}(1)=6\left(s_{3}(0)-\gamma\left(r_{3,0}\right)\right)=6, s_{3}(2)=6\left(s_{3}(1)-\gamma\left(r_{3,1}\right)\right)=6(6-1)=30, \ldots
$$


Let $k \geq 3$. We now turn to the general case to compute $\left(s_{k}(n)\right)_{n \geq 0}$. Consider the homomorphism of groups $\chi$ between $\left(\mathbf{H}_{k},+\right)$ and $\left(\mathbb{Z}\left(\left(z_{1}, \ldots, z_{k-1}\right)\right), \cdot\right)$ defined by the images of a basis of $\mathbf{H}_{k}$

$$
\chi: \mathbf{e}_{1}-\mathbf{e}_{k} \mapsto z_{1}, \mathbf{e}_{2}-\mathbf{e}_{k} \mapsto z_{2}, \ldots, \mathbf{e}_{k-1}-\mathbf{e}_{k} \mapsto z_{k-1}
$$

In particular, $\chi(-\mathbf{v})=1 / \chi(\mathbf{v})$ and $\chi\left(\mathbf{v}+\mathbf{v}^{\prime}\right)=\chi(\mathbf{v}) \cdot \chi\left(\mathbf{v}^{\prime}\right)$. Any element of $\mathbf{H}_{k}$ can be decomposed as a linear combination with integer coefficients of $\mathbf{e}_{1}-$ $\mathbf{e}_{k}, \mathbf{e}_{2}-\mathbf{e}_{k}, \ldots, \mathbf{e}_{k-1}-\mathbf{e}_{k}$ and corresponds by $\chi$ to a unique Laurent polynomial in $z_{1}, \ldots, z_{k-1}$. Each vertex $\mathbf{x}$ in $\mathbf{H}_{k}$ has exactly $k(k-1)$ neighbors:

$$
\mathbf{x}+\left\{\mathbf{e}_{i}-\mathbf{e}_{j} \mid 1 \leq i, j \leq k, i \neq j\right\}
$$

and these $k(k-1)$ translations of $\mathbf{x}$ are coded through $\chi$ by the terms

$$
\left\{z_{i}+\frac{1}{z_{i}} \mid 1 \leq i \leq k-1\right\} \cup\left\{\frac{z_{i}}{z_{j}} \mid 1 \leq i, j \leq k-1, i \neq j\right\} .
$$

Now consider the Laurent polynomial corresponding to these elementary translations:

$$
T=\sum_{i=1}^{k-1}\left(z_{i}+\frac{1}{z_{i}}\right)+\sum_{i \neq j} \frac{z_{i}}{z_{j}}=\left(1+\sum_{i=1}^{k-1} z_{i}\right)\left(1+\sum_{i=1}^{k-1} \frac{1}{z_{i}}\right)-k .
$$

Let $\mathbf{x} \in \mathbf{H}_{k}$ and $\left(j_{1}, \ldots, j_{k-1}\right) \in \mathbb{Z}^{k-1}$ be such that $\chi(\mathbf{x})=z_{1}^{j_{1}} \cdots z_{k-1}^{j_{k-1}}$. The number of paths of length $n$ from the origin to $\mathbf{x}$ in the lattice $\mathbf{H}_{k}$ is given by the coefficient of $z_{1}^{j_{1}} \cdots z_{k-1}^{j_{k-1}}$ in $T^{n}$. In particular, the constant term gives exactly the number of paths of length $n$ returning to the origin. Furthermore, for $k=3$ one can derive (2).

Example 3. For $k=4$, the number of paths of length $n$ in $\mathbf{H}_{4}$ starting and ending at the origin is Sloane's sequence A002899 and is given by

$$
\sum_{\ell=0}^{n}(-4)^{n-\ell}\left(\begin{array}{l}
n \\
\ell
\end{array}\right) \sum_{j=0}^{\ell}\left(\begin{array}{l}
\ell \\
j
\end{array}\right)^{2}\left(\begin{array}{c}
2 \ell-2 j \\
\ell-j
\end{array}\right)\left(\begin{array}{c}
2 j \\
j
\end{array}\right)
$$

The first values are $1,0,12,48,540,4320,42240,403200,4038300, \ldots$. For $k=$ 5 , we get $1,0,20,120,1860,23280,342200,5115600,79922500, \ldots$ and for $k=6$ : $1,0,30,240,4770,82080,1650900,34524000,758894850, \ldots$.

Being able to compute $\left(\mathcal{S}^{n} e\right)(\mathbf{0})$ for the lattice $\mathbf{H}_{k}$, we can proceed exactly as for the computation of $s_{3}(n)$ and get, for all $n \geq 0$,

$$
\gamma\left(r_{k, n+1}\right)=\frac{\left(\mathcal{S}^{n+2} e\right)(\mathbf{0})}{k(k-1)}-\sum_{i=0}^{n} \gamma\left(r_{k, i}\right)\left(\mathcal{S}^{n-i+1} e\right)(\mathbf{0})
$$

with $\gamma\left(r_{k, 0}\right)=0$ and finally, $s_{k}(n+1)=k(k-1)\left(s_{k}(n)-\gamma\left(r_{k, n}\right)\right)$. 


\section{About the Thue--Morse Word}

Currie and Saari $[4]$ proved that if $n \not \equiv 1(\bmod 6)$, then the Thue-Morse word $\mathbf{t}$ has an unbordered factor of length $n$, but they left it open to decide for which lengths congruent to 1 modulo 6 does this property hold. Then Goč, Henshall and Shallit [6] showed that $\mathbf{t}$ has an unbordered factor of length $n$ if and only if $(n)_{2} \notin 1\left(01^{*} 0\right)^{*} 10^{*} 1$, where $(n)_{2}$ denotes the base 2 expansion of $n$.

If we are interested in abelian unbordered factors of length $n$ occurring in $\mathbf{t}$, we obtain a strict subset of the set described by the above theorem. For instance, for $n=9,(n)_{2}=1001$ does not belong to $1\left(01^{*} 0\right)^{*} 10^{*} 1$, so $\mathbf{t}$ contains an unbordered factor of length 9 but a direct inspection shows that all factors of length 9 occurring in $\mathbf{t}$ are abelian bordered. For instance, the factor 001100101 is unbordered but is abelian bordered. Obtained by a computer search, the first few values of $n \in\{0, \ldots, 2000\}$ such that $\mathbf{t}$ has an abelian unbordered factor of length $n$ are $0,1,2,3,5,8,10,12,14,16,22,50,54,66,70,194,198,258,262$, $770,774,1026,1030$. We conjecture that the set of integers $n \geq 50$ such that $\mathbf{t}$ has an abelian unbordered factor of length $n$ consists of those integers whose base 2 expansion belongs to $110(00)^{*}\{01,11\} 0 \cup 10(00)^{+}\{01,11\} 0$.

Generally, abelian properties of $k$-automatic sequences are not suited to be expressed in the extended Presburger arithmetic $\left\langle\mathbb{N},+, V_{k}\right\rangle$. Nevertheless, we can take advantage of the fact that the Thue-Morse word is abelian periodic of period 2 and apply Büchi's theorem [2] with a technique similar to $[3,6]$. We take verbatim the statement of Büchi's theorem as formulated by Charlier, Rampersad and Shallit in [3], which states that the $k$-automatic sequences are exactly the sequences definable in the first order structure $\left\langle\mathbb{N},+, V_{k}\right\rangle$.

Theorem 1. [3] If we can express a property of a $k$-automatic sequence $\mathbf{x}$ using quantifiers, logical operations, integer variables, the operations of addition, subtraction, indexing into $\mathbf{x}$, and comparison of integers or elements of $\mathbf{x}$, then this property is decidable.

The technique we are now describing can obviously be adapted to any $k$ automatic abelian periodic word. We will give in (4) below a first order formula $\varphi(n)$ in $\left\langle\mathbb{N},+, V_{2}\right\rangle$ that is satisfied if and only if an abelian unbordered factor of length $n$ occurs in the Thue-Morse word $\mathbf{t}$. General procedures to obtain a finite automaton recognizing the base 2 expansions of the integers belonging to the set $\left\{n \in \mathbb{N} \mid\left\langle\mathbb{N},+, V_{2}\right\rangle \models \varphi(n)\right\}$ do exist (see for instance [2]). Hence a certified regular expression for the base 2 expansion of the elements in the above set will follow. Note that, since $\mathbf{t}$ is 2 -automatic, we can define in $\left\langle\mathbb{N},+, V_{2}\right\rangle$ a unary function that maps $i$ to $\mathbf{t}(i)$. Such a formula is again described in [2]. Predicates $e(n)$ and $o(n)$ are simply shorthands to characterize even and odd integers, $e(n) \equiv(\exists x)(n=x+x), o(n)=\neg e(n)$. We define a predicate $B(i, n, k)$ which is true if and only if the Thue-Morse word has an abelian bordered factor of length $n$ occurring at $i$ with a border of length $k$. Since the Thue-Morse word $\mathbf{t}$ is a concatenation of $a b$ and $b a$, discussing only the parity of the position $i$, the length $n$ of the factor and the length $k$ of the border, the predicate $B(i, n, k)$ is 
defined by the disjunction of the following terms $(e(i) \wedge e(n) \wedge e(k)),(e(i) \wedge e(n) \wedge$ $o(k) \wedge \mathbf{t}(i+k-1)=\mathbf{t}(i+n-k)),(e(i) \wedge o(n) \wedge e(k) \wedge \mathbf{t}(i+n-k) \neq \mathbf{t}(i+n-1))$, $(e(i) \wedge o(n) \wedge o(k) \wedge \mathbf{t}(i+k-1)=\mathbf{t}(i+n-1)),(o(i) \wedge e(n) \wedge o(k) \wedge \mathbf{t}(i)=\mathbf{t}(i+n-1))$, $(o(i) \wedge o(n) \wedge e(k) \wedge \mathbf{t}(i) \neq \mathbf{t}(i+k-1)),(o(i) \wedge o(n) \wedge o(k) \wedge \mathbf{t}(i)=\mathbf{t}(i+n-k))$ and $(o(i) \wedge e(n) \wedge e(k) \wedge[(\mathbf{t}(i)=\mathbf{t}(i+n-k) \wedge \mathbf{t}(i+k-1)=\mathbf{t}(i+n-1))$ $\vee(\mathbf{t}(i)=\mathbf{t}(i+n-1) \wedge \mathbf{t}(i+k-1)=\mathbf{t}(i+n-k))])$. As an example, if $i$ is even, $n$ and $k$ are odd, we have the situation depicted in Figure 4 . In that case, since all blocks $a b$ and $b a$ are abelian equivalent, one has just to check equality of two symbols in adequate positions corresponding to the parameters.

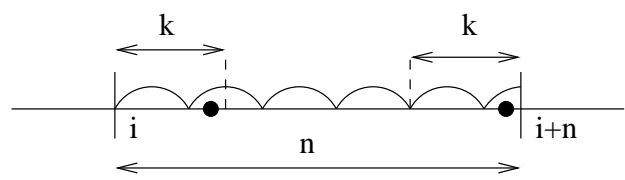

Fig. 4. A factor occurring in the Thue-Morse word.

Now the Thue-Morse word has an abelian unbordered factor of length $n$ if and only if the following formula holds true

$$
\varphi(n) \equiv(\exists i)(\forall k)(k \geq 1 \wedge 2 k \leq n) \rightarrow \neg B(i, n, k) .
$$

\section{References}

1. M. Barnabei, F. Bonetti, M. Silimbani, Restricted involutions and Motzkin paths, Adv. in Appl. Math. 47 (2011), 102-115.

2. V. Bruyère, G. Hansel, C. Michaux, R. Villemaire, Logic and p-recognizable sets of integers, Bull. Belg. Math. Soc 1 (1994), 191-238.

3. E. Charlier, N. Rampersad, J. Shallit, Enumeration and decidable properties of automatic sequences, Int. J. Found. Comput. Sci., 23 (2012), 1035-1066.

4. J. D. Currie, K. Saari, Least periods of factors of infinite words, RAIRO Inform. Théor. App. 43 (2009), 165-178.

5. A. Ehrenfeucht, D. M. Silberger, Periodicity and unbordered segments of words, Disc. Math. 26 (1979), 101-109.

6. D. Goč, D. Henshall, J. Shallit, Automatic theorem-proving in combinatorics on words. In CIAA 2012, Lect. Notes in Comp. Science 7381 (2012), 180-191.

7. D. Graham, D. E. Knuth, O. Patashnik, Concrete mathematics. A foundation for computer science, Second edition, Addison-Wesley Pub. Company, (1994).

8. T. Harju, D. Nowotka, Periodicity and Unbordered Words: A Proof of Duval's Conjecture, J. ACM $5 \mathbf{4}$ (2007).

9. S. Holub, K. Saari, On highly palindromic words, Disc. Appl. Math. 157 (2009), 953-959.

10. O. Guibert, E. Pergola, Enumeration of vexillary involutions which are equal to their mirror/complement, Disc. Math. 224 (2000), 281-287.

11. A. Sapounakis, P. Tsikouras, On $k$-colored Motzkin words, J. Integer Seq. 7 (2004).

12. Neil J. A. Sloane, The On-Line Encyclopedia of Integer Sequences, The OEIS Foundation Inc., http://oeis.org/. 